\title{
Local Derivative Pattern Versus Local Binary Pattern: Face Recognition With High-Order Local Pattern Descriptor
}

\author{
Baochang Zhang, Yongsheng Gao, Senior Member, IEEE, Sanqiang Zhao, and Jianzhuang Liu, Senior Member, IEEE
}

\begin{abstract}
This paper proposes a novel high-order local pattern descriptor, local derivative pattern (LDP), for face recognition. LDP is a general framework to encode directional pattern features based on local derivative variations. The $n^{t h}$-order LDP is proposed to encode the $(n-1)^{t h}$-order local derivative direction variations, which can capture more detailed information than the first-order local pattern used in local binary pattern (LBP). Different from LBP encoding the relationship between the central point and its neighbors, the LDP templates extract high-order local information by encoding various distinctive spatial relationships contained in a given local region. Both gray-level images and Gabor feature images are used to evaluate the comparative performances of LDP and LBP. Extensive experimental results on FERET, CAS-PEAL, CMU-PIE, Extended Yale B, and FRGC databases show that the high-order LDP consistently performs much better than LBP for both face identification and face verification under various conditions.
\end{abstract}

Index Terms-Face recognition, Gabor feature, high-order local pattern, local binary pattern (LBP), local derivative pattern (LDP).

\section{INTRODUCTION}

A good object representation or object descriptor is one of the key issues for a well-designed face recognition system [4], [32]. Representation issues include: what representation is desirable for the recognition of a pattern and how to effectively extract the representation from the original input image. An efficient descriptor should be of high ability to discriminate between classes, has low intraclass variance, and can be easily computed. Many holistic methods, such as Eigenface [24] and Fisherface [3] built on principal component analysis (PCA) and linear discriminant analysis (LDA) respectively, have been proved successful.

Manuscript received March 08, 2009; revised August 16, 2009. First published November 03, 2009; current version published January 15, 2010. This work was supported in part by the Australian Research Council (ARC) under Discovery Grants DP0451091 and DP0877929, and in part by the Natural Science Foundation of China under Grant 60903065 . The associate editor coordinating the review of this manuscript and approving it for publication was Dr. Laurent Younes.

B. Zhang is with the School of Automation Science and Electrical Engineering, Beihang University, Beijing 100191, China (e-mail: bczhang@buaa. edu.cn).

Y. Gao and S. Zhao are with the Griffith School of Engineering, Griffith University, Nathan Campus, Brisbane, QLD 4111, Australia (e-mail: yongsheng. gao@griffith.edu.au; s.zhao@griffith.edu.au).

J. Liu is with the Department of Information Engineering, The Chinese University of Hong Kong, Hong Kong (e-mail: jzliu@ie.cuhk.edu.hk).

Color versions of one or more of the figures in this paper are available online at http://ieeexplore.ieee.org.

Digital Object Identifier 10.1109/TIP.2009.2035882
Recently, local descriptors have gained much attention in the face recognition community for their robustness to illumination and pose variations. One of the local descriptors is local feature analysis (LFA) proposed by Penev et al. [18]. In LFA, a dense set of local-topological fields are developed to extract local features. Through discovering a description of one class objects with the derived local features, LFA is a purely second-order statistic method. Gabor wavelet is a sinusoidal plane wave with particular frequency and orientation, modulated by a Gaussian envelope [6]. It can characterize the spatial structure of an input object, and thus is suitable for extracting local features. Elastic Bunch Graph Matching (EBGM) [27] represents a face by a topological graph where each node contains a group of Gabor coefficients, known as a jet. It achieves a noticeable performance in the FERET test [20]. The feasibility of the component or patch based face recognition is also investigated in [12], in which the component-based face recognition approaches clearly outperform holistic approaches.

The recently proposed local binary pattern (LBP) features are originally designed for texture description [16], [17], [21]. The operator has been successfully applied to facial expression analysis [31], background modeling [11] and face recognition [1]. In face recognition, it achieves a much better performance than Eigenface, Bayesian and EBGM methods, providing a new way of investigating into the face representation. The idea behind using the LBP features is that a face can be seen as a composition of micropatterns [1]. LBP in nature represents the first-order circular derivative pattern of images, a micropattern generated by the concatenation of the binary gradient directions. However, the first-order pattern fails to extract more detailed information contained in the input object. To the best of our knowledge, no high-order local pattern operator has been investigated for face representation. In fact, the high-order operator can capture more detailed discriminative information. Some high-order nonlocal pattern methods have been successfully used to solve the face recognition problem. The PCA representation can hardly capture some variations in the training dataset, such as pose in face recognition. Independent Component Analysis (ICA) takes higher-order statistics into account, and is suitable for learning complex structure in the dataset [2], [13]. In [10], 25 local autocorrelation coefficients are exploited to calculate the high-order primitive features, which are further combined with LDA and appear robust against changes in facial expression. We can also find other high-order techniques used in face recognition such as the mutual information for feature selection [22], in which high-order statistic method is used to select more discriminative 
features. In Tensorface [26], the algebra of higher-order tensors offers a potent mathematical framework for analyzing ensembles of faces resulting from the interaction of any number of underlying factors. The feasibility of a high-order neural network is also investigated in [25].

In this paper, we propose a novel object descriptor, the highorder Local Derivative Pattern (LDP), for robust face recognition. In our framework, LBP can be conceptually considered as a nondirectional first-order local pattern, which is the binary result of the first-order derivative in images. The second-order LDP can capture the change of derivative directions among local neighbors, and encode the turning point in a given direction. The $n^{\text {th }}$-order LDP is a local pattern presented in a general form which captures detailed relationship in a local neighborhood. Compared to LBP, the high-order LDP achieved superior performance in our comparative experiments. Moreover, we propose to extend LDP to feature images, Gabor real and imaginary features, for face recognition, which can effectively enhance the performance of the proposed LDP method, and LBP as well. Different from the learning-based approaches, LDP features are directly extracted from gray-level images or feature images without any training procedure. Like LBP, LDP is a micropattern representation which can also be modeled by histogram to preserve the information about the distribution of the LDP micropatterns.

The remaining part of this paper is organized as follows. Section II introduces and discusses the high-order LDP in detail. Section III extends LDP to the feature domain. In Section IV, extensive experiments on FERET [20], CAS-PEAL [7], CMU-PIE [23], Extended Yale B [9], [14], and FRGC [19] databases are conducted to evaluate the performance of the proposed method on face recognition. Finally, conclusions are drawn in Section V with some discussions.

\section{High-ORDER LOCAL PATTERN}

In this section, we provide a brief review of local binary pattern (LBP), and then introduce the second-order local derivative pattern (LDP) to calculate the first-order derivative direction variation. After that, the definition and feasibility of the general $n^{\text {th }}$-order LDP are presented and discussed. Finally, the spatial histogram is described for modeling the distribution of LDP of a face.

\section{A. Local Binary Pattern}

Derived from a general definition of texture in a local neighborhood, LBP is defined as a grayscale invariant texture measure and is a useful tool to model texture images. LBP later has shown excellent performance in many comparative studies, in terms of both speed and discrimination performance [1], [11], [17], [31]. The original LBP operator labels the pixels of an image by thresholding the $3 \times 3$ neighborhood of each pixel with the value of the central pixel and concatenating the results binomially to form a number. The thresholding function $f(\cdot, \cdot)$ for the basic LBP can be formally represented as

$$
\begin{aligned}
f & \left(I\left(Z_{0}\right), I\left(Z_{i}\right)\right) \\
& =\left\{\begin{array}{ll}
0, & \text { if } I\left(Z_{i}\right)-I\left(Z_{0}\right) \leq \text { threshold } \\
1, & \text { if } I\left(Z_{i}\right)-I\left(Z_{0}\right)>\text { threshold }
\end{array}, i=1,2, \cdots, 8\right.
\end{aligned}
$$

\begin{tabular}{|l|l|l|}
\hline$Z_{1}$ & $Z_{2}$ & $Z_{3}$ \\
\hline$Z_{8}$ & $Z_{0}$ & $Z_{4}$ \\
\hline$Z_{7}$ & $Z_{6}$ & $Z_{5}$ \\
\hline
\end{tabular}

Fig. 1. Example of 8-neighborhood around $Z_{0}$.

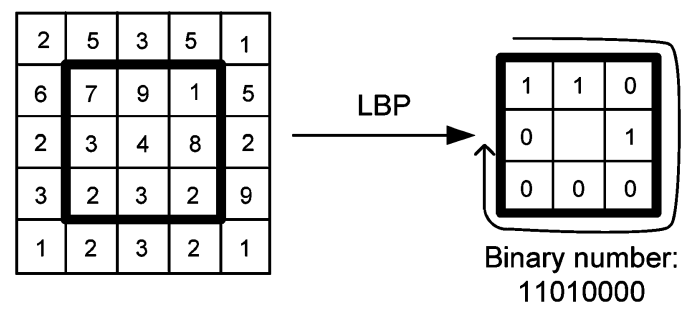

Fig. 2. Example of obtaining the LBP micropattern for the region in the black square.

where $Z_{i}, i=1, \cdots, 8$ is an 8 -neighborhood point around $Z_{0}$ as shown in Fig. 1. An LBP can also be considered as the concatenation of the binary gradient directions, and is called a micropattern. Fig. 2 shows an example of obtaining an LBP micropattern when the threshold is set to zero. The histograms of these micropatterns contain information of the distribution of the edges, spots, and other local features in an image. LBP has been successfully used for face recognition [1]. Different from statistic learning methods tuning a large number of parameters, the LBP method is very efficient due to its easy-to-compute feature extraction operation and simple matching strategy.

\section{B. Local Derivative Pattern}

LBP actually encodes the binary result of the first-order derivative among local neighbors by using a simple threshold function as shown in (1), which is incapable of describing more detailed information. In this paper, we investigate the feasibility and effectiveness of using high-order local patterns for face representation. An LDP operator is proposed, in which the $(n-1)^{t h}$-order derivative direction variations based on a binary coding function. In this scheme, LBP is conceptually regarded as the nondirectional first-order local pattern operator, because LBP encodes all-direction first-order derivative binary result while LDP encodes the higher-order derivative information which contains more detailed discriminative features that the first-order local pattern (LBP) can not obtain from an image.

Given an image $I(Z)$, the first-order derivatives along $0^{\circ}$, $45^{\circ}, 90^{\circ}$ and $135^{\circ}$ directions are denoted as $I_{\alpha}^{\prime}(Z)$ where $\alpha=$ $0^{\circ}, 45^{\circ}, 90^{\circ}$ and $135^{\circ}$. Let $Z_{0}$ be a point in $I(Z)$, and $Z_{i}, i=$ $1, \cdots, 8$ be the neighboring point around $Z_{0}$ (see Fig. 1). The four first-order derivatives at $Z=Z_{0}$ can be written as

$$
\begin{aligned}
I_{0^{\circ}}^{\prime}\left(Z_{0}\right) & =I\left(Z_{0}\right)-I\left(Z_{4}\right) \\
I_{45^{\circ}}^{\prime}\left(Z_{0}\right) & =I\left(Z_{0}\right)-I\left(Z_{3}\right) \\
I_{90^{\circ}}^{\prime}\left(Z_{0}\right) & =I\left(Z_{0}\right)-I\left(Z_{2}\right) \\
I_{135^{\circ}}^{\prime}\left(Z_{0}\right) & =I\left(Z_{0}\right)-I\left(Z_{1}\right) .
\end{aligned}
$$




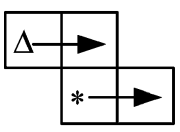

(a-1)

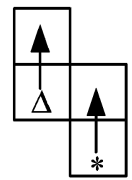

(c-1)

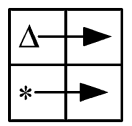

$(\mathrm{a}-2)$

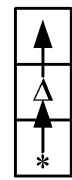

(c-2)

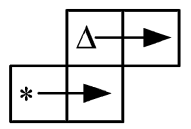

(a-3)

(a)

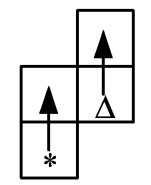

(c-3)

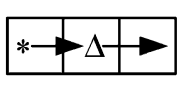

(a-4)

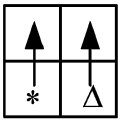

(c-4)

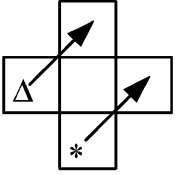

(b-1)

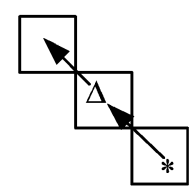

$(d-1)$

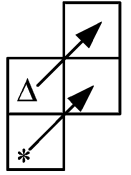

(b-2)

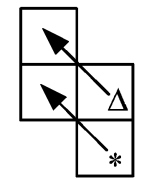

(d-2)

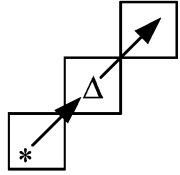

(b-3)

(b)

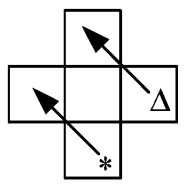

(d-3)

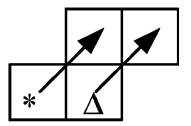

(b-4)

(c)

Fig. 3. Illustration of LDP templates. (a-1) The template for calculating $f\left(I_{0^{\circ}}^{n-1}\left(Z_{0}\right), I_{0^{\circ}}^{n-1}\left(Z_{1}\right)\right)$ and $f\left(I_{0^{\circ}}^{n-1}\left(Z_{0}\right), I_{0 \circ}^{n-1}\left(Z_{5}\right)\right)$. (a-2) The template for calculating $f\left(I_{0 \circ}^{n-1}\left(Z_{0}\right), I_{0 \circ}^{n-1}\left(Z_{2}\right)\right)$ and $f\left(I_{0^{\circ}}^{n-1}\left(Z_{0}\right), I_{0 \circ}^{n-1}\left(Z_{6}\right)\right)$. (a-3) The template for calculating $f\left(I_{0^{\circ}}^{n-1}\left(Z_{0}\right), I_{0^{\circ}}^{n-1}\left(Z_{3}\right)\right)$ and $f\left(I_{0^{\circ}}^{n-1}\left(Z_{0}\right), I_{0^{\circ}}^{n-1}\left(Z_{7}\right)\right)$. (a-4) The template for calculating $f\left(I_{0^{\circ}}^{n-1}\left(Z_{0}\right), I_{0^{\circ}}^{n-1}\left(Z_{4}\right)\right)$ and $f\left(I_{0^{\circ}}^{n-1}\left(Z_{0}\right), I_{0^{\circ}}^{n-1}\left(Z_{8}\right)\right)$. (b-1) The template for calculating $f\left(I_{45^{\circ}}^{n-1}\left(Z_{0}\right), I_{45^{\circ}}^{n-1}\left(Z_{1}\right)\right)$ and $f\left(I_{450}^{n-1}\left(Z_{0}\right), I_{450}^{n-1}\left(Z_{5}\right)\right)$. (b-2) The template for calculating $f\left(I_{45^{\circ}}^{n}{ }^{\circ}\left(Z_{0}\right), I_{45^{\circ}}^{n-1}\left(Z_{2}\right)\right)$ and $f\left(I_{450}^{n-1}\left(Z_{0}\right), I_{450}^{n-1}\left(Z_{6}\right)\right)$. (b-3) The template for calculating $f\left(I_{45^{\circ}}^{n-1}\left(Z_{0}\right), I_{45^{\circ}}^{n-1}\left(Z_{3}\right)\right)$ and $f\left(I_{45^{\circ}}^{n-1}\left(Z_{0}\right), I_{45^{\circ}}^{n-1}\left(Z_{7}\right)\right)$. (b-4) The template for calculating $f\left(I_{45^{\circ}}^{n-1}\left(Z_{0}\right), I_{45^{\circ}}^{n-1}\left(Z_{4}\right)\right)$ and $f\left(I_{45^{\circ}}^{n-1}\left(Z_{0}\right), I_{45^{\circ}}^{n-1}\left(Z_{8}\right)\right)$. (c-1) The template for calculating $f\left(I_{00^{\circ}}^{n n}\left(Z_{0}\right), I_{900}^{n-1}\left(Z_{1}\right)\right)$ and $f\left(I_{000}^{n-1}\left(Z_{0}\right), I_{000}^{n-1}\left(Z_{5}\right)\right)$. (c-2) The template for calculating $f\left(I_{000}^{n-1}\left(Z_{0}\right), I_{900}^{n-1}\left(Z_{2}\right)\right)$ and $f\left(I_{90^{\circ}}^{n-1}\left(Z_{0}\right), I_{90^{\circ}}^{n-1}\left(Z_{6}\right)\right)$. (c-3) The template for calculating $f\left(I_{900^{\circ}}^{n-1}\left(Z_{0}\right), I_{90^{\circ}}^{n-1}\left(Z_{3}\right)\right)$ and $f\left(I_{900^{\circ}}^{n-1}\left(Z_{0}\right), I_{90^{\circ}}^{n-1}\left(Z_{7}\right)\right)$. (c-4) The template for calculating $f\left(I_{90^{\circ}}^{n-1}\left(Z_{0}\right), I_{90^{\circ}}^{n-1}\left(Z_{4}\right)\right)$ and $f\left(I_{90^{\circ}}^{n-1}\left(Z_{0}\right), I_{90^{\circ}}^{n-1}\left(Z_{8}\right)\right)$. (d-1) The template for calculating $f\left(I_{135^{\circ}}^{n-1}\left(Z_{0}\right), I_{135^{\circ}}^{n-1}\left(Z_{1}\right)\right)$ and $f\left(I_{135^{\circ}}^{n-1}\left(Z_{0}\right), I_{135^{\circ}}^{n-1}\left(Z_{5}\right)\right)$. (d-2) The template for calculating $f\left(I_{135^{\circ}}^{n-1}\left(Z_{0}\right), I_{135^{\circ}}^{n-1}\left(Z_{2}\right)\right)$ and $f\left(I_{135^{\circ}}^{n-1}\left(Z_{0}\right), I_{135^{\circ}}^{n-1}\left(Z_{6}\right)\right)$. (d-3) The template for calculating $f\left(I_{135^{\circ}}^{n-1}\left(Z_{0}\right), I_{135^{\circ}}^{n-1}\left(Z_{3}\right)\right)$ and $f\left(I_{135^{\circ}}^{n-1}\left(Z_{0}\right), I_{135^{\circ}}^{n-1}\left(Z_{7}\right)\right)$. (d-4) The template for calculating $f\left(I_{135^{\circ}}^{n-1}\left(Z_{0}\right), I_{135^{\circ}}^{n-1}\left(Z_{4}\right)\right)$ and $f\left(I_{135^{\circ}}^{n-1}\left(Z_{0}\right), I_{135^{\circ}}^{n-1}\left(Z_{8}\right)\right)$. ref 1 and ref 2 are the reference points to be aligned to the point of $Z_{0}$. (a) $\alpha=0^{\circ}$, ref $1=*$, ref $2=\Delta$; (b) $\alpha=45^{\circ}$, ref $1=*$, ref $2=\Delta$; (c) $\alpha=90^{\circ}$, ref $1=*$, ref $2=\Delta$; (d) $\alpha=135^{\circ}$, ref $1=*$, ref $2=\Delta$.

The second-order directional LDP, $L D P_{\alpha}^{2}\left(Z_{0}\right)$, in $\alpha$ direction at $Z=Z_{0}$ is defined as

$$
\begin{array}{r}
L D P_{\alpha}^{2}\left(Z_{0}\right)=\left\{f\left(I_{\alpha}^{\prime}\left(Z_{0}\right), I_{\alpha}^{\prime}\left(Z_{1}\right)\right), f\left(I_{\alpha}^{\prime}\left(Z_{0}\right), I_{\alpha}^{\prime}\left(Z_{2}\right)\right)\right. \\
\left.\cdots, f\left(I_{\alpha}^{\prime}\left(Z_{0}\right), I_{\alpha}^{\prime}\left(Z_{8}\right)\right)\right\}
\end{array}
$$

where $f(\cdot, \cdot)$ is a binary coding function determining the types of local pattern transitions. It encodes the co-occurrence of two derivative directions at different neighboring pixels as

$$
f\left(I_{\alpha}^{\prime}\left(Z_{0}\right), I_{\alpha}^{\prime}\left(Z_{i}\right)\right)=\left\{\begin{array}{rr}
0, & \text { if } I_{\alpha}^{\prime}\left(Z_{i}\right) \cdot I_{\alpha}^{\prime}\left(Z_{0}\right)>0 \\
1, & \text { if } I_{\alpha}^{\prime}\left(Z_{i}\right) \cdot I_{\alpha}^{\prime}\left(Z_{0}\right) \leq 0 \\
i=1,2, \cdots, 8 .
\end{array}\right.
$$

Finally, the second-order Local Derivative Pattern, $L D P^{2}(Z)$, is defined as the concatenation of the four 8-bit directional LDPs

$$
L D P^{2}(Z)=\left\{L D P_{\alpha}^{2}(Z) \mid \alpha=0^{\circ}, 45^{\circ}, 90^{\circ}, 135^{\circ}\right\}
$$

It can be seen from the above equations that the proposed LDP operator labels the pixels of an image by comparing two derivative directions at two neighboring pixels and concatenating the results as a 32-bit binary sequence. The derivative direction comparisons defined in (7) are performed on 16 templates (Fig. 3) reflecting various distinctive spatial relationships in a local region. Different from LBP encoding the binary derivative gradient directions, the second-order LDP encodes the change of the neighborhood derivative directions, which represents the second-order pattern information in the local region.

Fig. 4 illustrates the types of local pattern transitions in an LDP template that are encoded into " 1 " and "0", respectively. Each of the 16 LDP templates in Fig. 3 can be classified as either a 3-point template or a 4-point template. For a 3-point template, (7) assigns a " 0 " to a monotonically increasing or decreasing pattern [see Fig. 4(a-2)], while a "turning point" pattern is labeled as a "1" [see Fig. 4(a-1)]. Similarly, for a 4-point template, a "gradient turning" pattern [see Fig. 4(b-1)] is labeled as a "1" and monotonically increasing or decreasing pattern is labeled as a "0" [see Fig. 4(b-2)]. This operator extracts higher-order local pattern information, i.e., the changes of first-order derivative direction information, into a binary string.

An example of the second-order LDP computation is illustrated in Fig. 5. To calculate the second-order directional Local Derivative Pattern, $L D P_{\alpha}^{2}\left(Z_{0}\right)$, in $\alpha=0^{\circ}$ direction at $Z=Z_{0}$, the four templates in Fig. 3(a) are applied on the image by aligning ref 1 and ref 2 to $Z_{0}$, respectively. When applying Template (a-1) by aligning ref 1 to $Z_{0}$, the two derivative directions defined by the two arrows in the template are monotonically increasing as shown by the left case in Fig. 4(b-2). Thus, "0" is assigned to this bit. Similarly, applying Templates (a-2), (a-3), and (a-4) with ref 1 aligned to $Z_{0}$, the two derivative directions defined by the two arrows in the templates are indicated by the left case in Fig. 4(b-1), the left case in Fig. 4(b-2), and the right case in Fig. 4(a-1), creating "101" for the next three bits. Repeat the above procedure with the same four templates by aligning ref 2 to $Z_{0}$, we can get " 0100 " for the last 4 bits of the 8-bit $L D P_{0^{\circ}}^{2}\left(Z_{0}\right)$. Now, we have " 01010100 " for the $0^{\circ}$ direction. In the same way, templates in Fig. 3(b)-(d) are applied on the image in Fig. 5 to obtain $L D P_{\alpha}^{2}\left(Z_{0}\right)$ in $\alpha=$ $45^{\circ}, 90^{\circ}$ and $135^{\circ}$ directions, respectively (see $L D P_{45^{\circ}}^{2}\left(Z_{0}\right)$, $L D P_{90^{\circ}}^{2}\left(Z_{0}\right)$ and $L D P_{135^{\circ}}^{2}\left(Z_{0}\right)$ in Fig. 5). Finally, a 32-bit $\operatorname{LDP}^{2}\left(Z_{0}\right)=01010100001011111101000011000110$ is generated by concatenating the four 8-bit directional LDPs as defined in (8). 

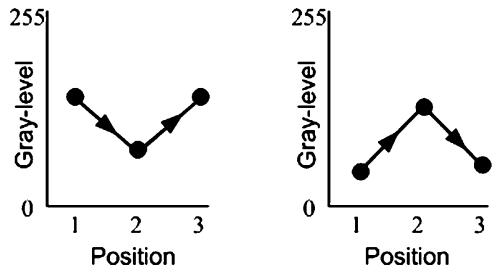

$(a-1)$

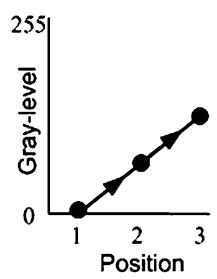

$(a-2)$

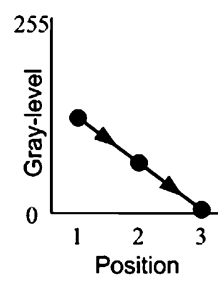

(a)
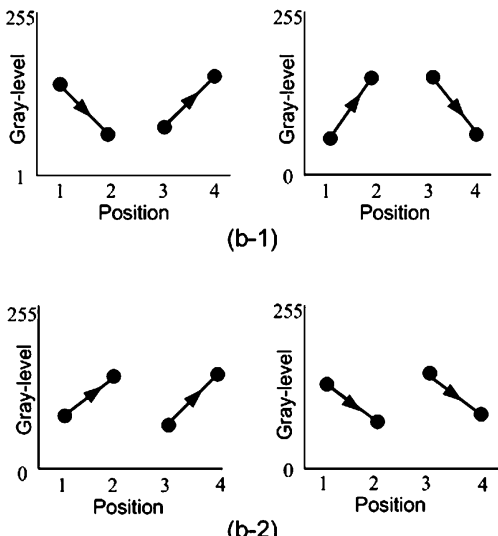

(b)

Fig. 4. Meanings of " 0 " and "1" for the second-order LDP. (a) The three points LDP template: both cases in (a-1) result in a "1"; both cases in (a-2) result in a " 0 ". (b) The four points LDP template: both cases in (b-1) result in a " 1 "; both cases in (b-2) result in a " 0 ".

\section{C. $N^{\text {th }}$-Order Local Derivative Pattern}

To calculate the third-order Local Derivative Pattern, we first compute the second-order derivatives along $0^{\circ}, 45^{\circ}, 90^{\circ}$ and $135^{\circ}$ directions, denoted as $I_{\alpha}^{\prime \prime}(Z)$ where $\alpha=0^{\circ}, 45^{\circ}, 90^{\circ}$, $135^{\circ}$. The third-order Local Derivative Pattern, $L D P_{\alpha}^{3}\left(Z_{0}\right)$, in $\alpha$ direction at $Z=Z_{0}$ is defined as

$$
\begin{array}{r}
L D P_{\alpha}^{3}\left(Z_{0}\right)=\left\{f\left(I_{\alpha}^{\prime \prime}\left(Z_{0}\right), I_{\alpha}^{\prime \prime}\left(Z_{1}\right)\right), f\left(I_{\alpha}^{\prime \prime}\left(Z_{0}\right), I_{\alpha}^{\prime \prime}\left(Z_{2}\right)\right)\right. \\
\left.\cdots, f\left(I_{\alpha}^{\prime \prime}\left(Z_{0}\right), I_{\alpha}^{\prime \prime}\left(Z_{8}\right)\right)\right\} .
\end{array}
$$

In a general formulation, the $n^{\text {th }}$-order LDP is a binary string describing gradient trend changes in a local region of directional $(n-1)^{t h}$-order derivative images $I_{\alpha}^{n-1}(Z)$ as

$$
\begin{aligned}
\operatorname{LDP}_{\alpha}^{n}\left(Z_{0}\right)=\{ & f\left(I_{\alpha}^{n-1}\left(Z_{0}\right), I_{\alpha}^{n-1}\left(Z_{1}\right)\right) \\
& f\left(I_{\alpha}^{n-1}\left(Z_{0}\right), I_{\alpha}^{n-1}\left(Z_{2}\right)\right), \\
& \left.\cdots, f\left(I_{\alpha}^{n-1}\left(Z_{0}\right), I_{\alpha}^{n-1}\left(Z_{8}\right)\right)\right\}
\end{aligned}
$$

where $I_{\alpha}^{n-1}\left(Z_{0}\right)$ is the $(n-1)^{t h}$-order derivative in $\alpha$ direction at $Z=Z_{0} \cdot f\left(I_{\alpha}^{n-1}\left(Z_{0}\right), I_{\alpha}^{n-1}\left(Z_{i}\right)\right)$ is defined in (11), which encodes the $(n-1)^{t h}$-order gradient transitions into binary patterns, providing an extra order pattern information on the local region

$$
\begin{aligned}
& f\left(I_{\alpha}^{n-1}\left(Z_{0}\right), I_{\alpha}^{n-1}\left(Z_{i}\right)\right) \\
& =\left\{\begin{array}{ll}
0, & \text { if } I_{\alpha}^{n-1}\left(Z_{i}\right) \cdot I_{\alpha}^{n-1}\left(Z_{0}\right)>0 \\
1, & \text { if } I_{\alpha}^{n-1}\left(Z_{i}\right) \cdot I_{\alpha}^{n-1}\left(Z_{0}\right) \leq 0
\end{array}, \quad i=1,2, \cdots 8 .\right.
\end{aligned}
$$

The high-order local patterns provide a stronger discriminative capability in describing detailed texture information than the first-order local pattern as used in LBP. However, they tend to be sensitive to noise when the order $n$ becomes high. In the design of the proposed approach, the last-order operation (11) only preserves the coarse gradient direction transition information instead of conventional difference information. This can alleviate the noise sensitivity problem in the high-order LDP representation, making it more robust and stable in binary encoding identity patterns in human faces. In representing and recognizing many objects, such as human faces, the relative orientation information of each local region with respect to the object as a whole is part of the object identity patterns. This is particularly important in designing a highly discriminative object descriptor for distinguishing similar objects. In face recognition, for example, it has been demonstrated in [8] that edge orientations provide strong identity descriptive capability for classification. Even the average orientation value of a face Line Edge Map (LEM) [8] contains identity information, which can be used for face prefiltering. Therefore, the proposed LDP builds upon directional derivatives, without losing generality of the method, in four directions with a $45^{\circ}$ representation resolution. Other numbers of directions can also be used. The $n^{\text {th }}$-order LDP is a local pattern string defined as

$$
L D P^{n}(Z)=\left\{L D P_{\alpha}^{n}(Z) \mid \alpha=0^{\circ}, 45^{\circ}, 90^{\circ}, 135^{\circ}\right\} .
$$

It labels each pixel of the image with a 32-bit binary string encoding local texture pattern around the pixel in 16 measuring templates as illustrated in Fig. 3.

In calculating the $L D P^{n}(Z)$ of a given image $I(Z)$, the above binary pattern encoding process can be illustrated using the LDP template in Fig. 3, and the binary coding depicted in Fig. 4. Applying the template in Fig. 3(a-1) on $I_{\alpha}^{n-2}(Z)$ by aligning the reference point ref 1 to the point $\left(Z=Z_{0}\right)$ to be computed, the first encoded bit of $L D P^{n}\left(Z_{0}\right)$ is assigned " 0 " if the changes of $I_{0^{\circ}}^{n-2}(Z)$ enclosed in the template of Fig. 3(a-1) along the two arrows are as Fig. 4(b-2), and "1" otherwise. Similarly, the second, third and fourth bits of $L D P^{n}\left(Z_{0}\right)$ are labeled using the LDP templates (a-2) to (a-4) in Fig. 3, respectively, by aligning the reference point ref 1 to $Z_{0}$ in $I_{0^{\circ}}^{n-2}(Z)$. The bits 5 to 8 of $L D P^{n}\left(Z_{0}\right)$ are determined using the LDP templates (a-1) to (a-4) in Fig. 3, respectively, one more time by aligning the reference point to ref 2 to $Z_{0}$. This first 8 bits of $L D P^{n}\left(Z_{0}\right)$ is from the $\mathrm{n}^{\text {th }}$-order LDP in $0^{\circ}$ direction, $L D P_{0^{\circ}}^{n}\left(Z_{0}\right)$. The rest of the $3 \times 8$ bits of $L D P^{n}\left(Z_{0}\right)$ can be determined in the same way by applying the LDP templates (b-1)-(b-4), (c-1)-(c-4), and (d-1)-(d-4) to $I_{45^{\circ}}^{n-2}\left(Z_{0}\right), I_{90^{\circ}}^{n-2}\left(Z_{0}\right)$ and $I_{135^{\circ}}^{n-2}\left(Z_{0}\right)$, respectively. Fig. 6 provides visualized examples of LBP and LDP representations 


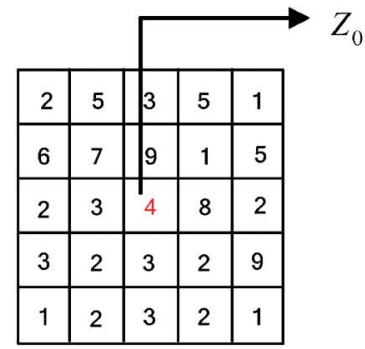

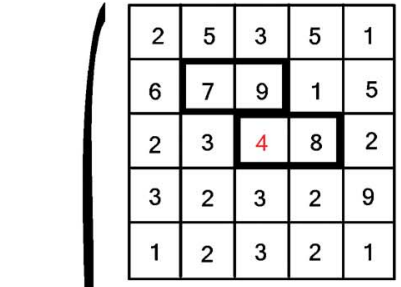

Template (a-1) ref $1=Z_{0}$ bit $=0$

$0^{\circ}$

\begin{tabular}{|l|l|l|l|l|}
\hline 2 & 5 & 3 & 5 & 1 \\
\hline 6 & 7 & 9 & 1 & 5 \\
\hline 2 & 3 & 4 & 8 & 2 \\
\hline 3 & 2 & 3 & 2 & 9 \\
\hline 1 & 2 & 3 & 2 & 1 \\
\hline
\end{tabular}

Template (a-1) ref $2=Z_{0}$ bit $=0$

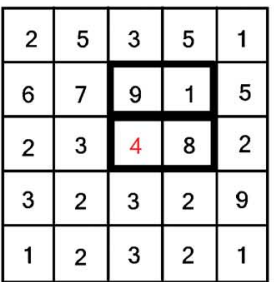

Template (a-2)

ref $1=Z_{0}$

bit $=1$

\begin{tabular}{|l|l|l|l|l|}
\hline 2 & 5 & 3 & 5 & 1 \\
\hline 6 & 7 & 9 & 1 & 5 \\
\hline 2 & 3 & 4 & 8 & 2 \\
\hline 3 & 2 & 3 & 2 & 9 \\
\hline 1 & 2 & 3 & 2 & 1 \\
\hline
\end{tabular}

Template (a-2)

ref $2=Z_{0}$

bit $=1$

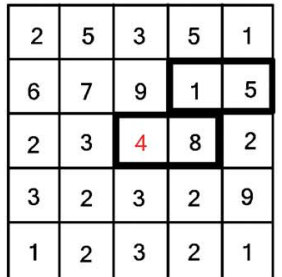

Template (a-3)

ref $1=Z_{0}$

bit $=0$

\begin{tabular}{|l|l|l|l|l|}
\hline 2 & 5 & 3 & 5 & 1 \\
\hline 6 & 7 & 9 & 1 & 5 \\
\hline 2 & 3 & 4 & 8 & 2 \\
\hline 3 & 2 & 3 & 2 & 9 \\
\hline 1 & 2 & 3 & 2 & 1 \\
\hline
\end{tabular}

Template (a-3)

ref $2=Z_{0}$

bit $=0$

\begin{tabular}{|l|l|l|l|l|}
\hline 2 & 5 & 3 & 5 & 1 \\
\hline 6 & 7 & 9 & 1 & 5 \\
\hline 2 & 3 & 4 & 8 & 2 \\
\hline 3 & 2 & 3 & 2 & 9 \\
\hline 1 & 2 & 3 & 2 & 1 \\
\hline
\end{tabular}

Template (a-4)

ref $1=Z_{0}$

bit $=1$

\begin{tabular}{|l|l|l|l|l|}
\hline 2 & 5 & 3 & 5 & 1 \\
\hline 6 & 7 & 9 & 1 & 5 \\
\hline 2 & 3 & 4 & 8 & 2 \\
\hline 3 & 2 & 3 & 2 & 9 \\
\hline 1 & 2 & 3 & 2 & 1 \\
\hline
\end{tabular}

Template (a-4)

ref $2=Z_{0}$

bit $=0$

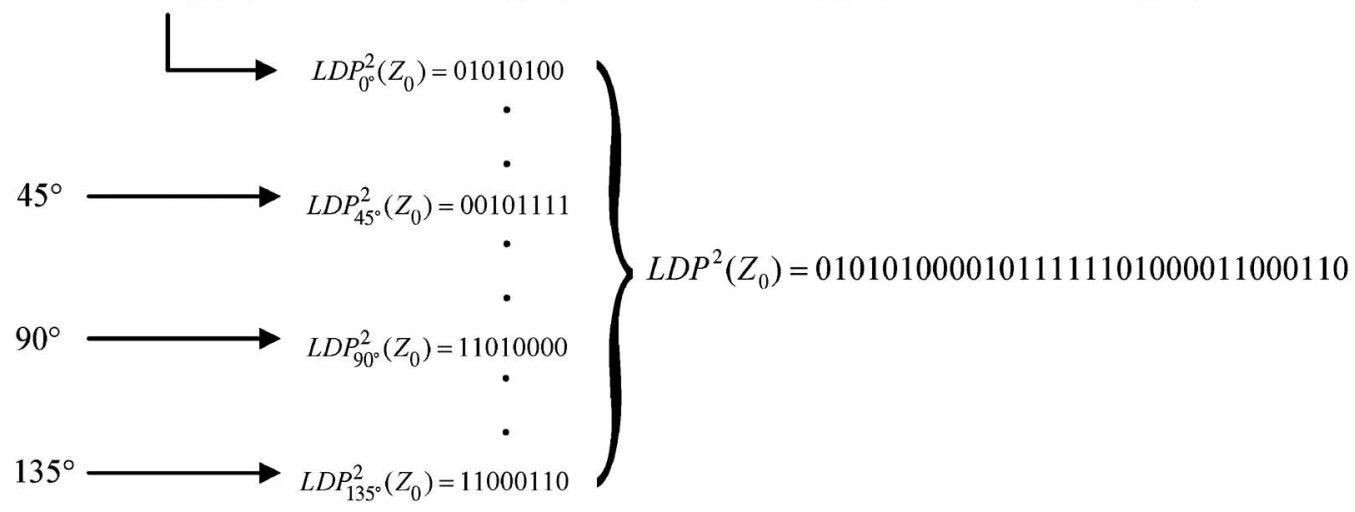

Fig. 5. Example to obtain the second-order LDP micropatterns.

for a face image, showing that the high-order LDP can capture more detailed information.

As we have demonstrated in Fig. 6, the LDP operator extracts detailed high-order information. The higher the order is, the more details the local pattern operator can extract from the image. This can be visually observed from Fig. 6 that as the operator order increases from the first-order [see Fig. 6(b)] to the second order [see Fig. 6(c)], the third order [see Fig. 6(d)], and up to the fourth order [see Fig. 6(e)], more and more details are extracted from the image. However, over-detailed patterns tend to be noise instead of identity information. When LDP reaches to the fourth order, more noise is extracted while identity facial features become indiscernible as can be seen in Fig. 6(e). This explains why the performance of LDP drops when it reaches to the fourth order. It should be noted that the experimental results demonstrated that both the second and third-order LDPs perform consistently superior to the first-order LBP, indicating that the second and third-order LDPs can better capture more detailed discriminative information than LBP for face recognition.

The advantages of the high-order LDP over LBP can be briefly summarized in the following aspects.

1) LBP cannot provide a detailed description for faces by encoding the binary gradient directions. However, the $n^{\text {th }}$-order LDP can provide more detailed description by coding the $(n-1)^{t h}$-order derivative direction variations. 


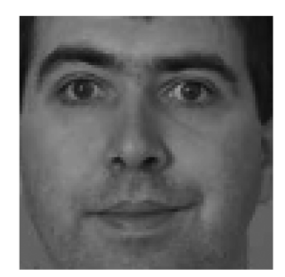

(a)

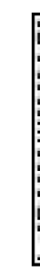

(c)

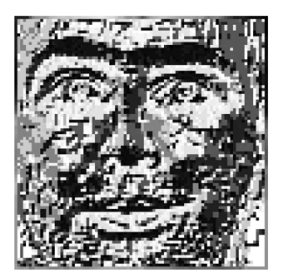

(b)

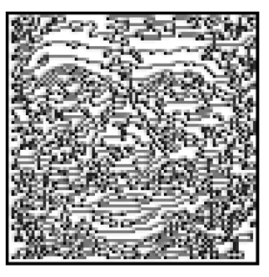

(d) (e)

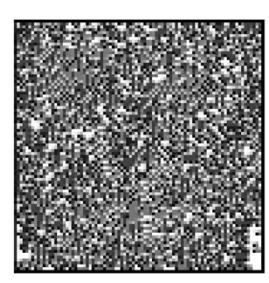

Fig. 6. Visualization of LBP and LDP (in $0^{\circ}$ direction) representations. (a) Original face image. (b) LBP. (c) The second-order LDP. (d) The third-order LDP. (e) The fourth-order LDP.

2) LBP encodes the relationship between the central point and its neighbors, but LDP encodes the various distinctive spatial relationships in a local region and, therefore, contains more spatial information.

\section{Histogram of Local Derivative Pattern}

In this paper, the LDP method presented in the above subsection is used for face representation. The procedure applies a high-order local feature operator on each pixel to extract discriminative features from its neighborhood. We model the distribution of high-order local derivative pattern by spatial histogram [1], [30], because it is more robust against variations in pose or illumination than holistic methods [1]. Given a direction $\alpha$, $L D P_{\alpha}$ are spatially divided into rectangular regions represented by $R_{1}, \cdots, R_{L}$, from which spatial histograms $\operatorname{HLDP}(i, \alpha)$ are extracted as

$$
\begin{aligned}
H L D P(i, \alpha)=\left\{H_{L D P_{\alpha}}\left(R_{i}\right) \mid i\right. & =1, \cdots, L ; \\
\alpha & \left.=0^{\circ}, 45^{\circ}, 90^{\circ}, 135^{\circ}\right\}
\end{aligned}
$$

where $H_{L D P_{\alpha}}\left(R_{i}\right)$ is the LDP histogram feature extracted from the local region $R_{i}$. Note that the regions do not have to be rectangular or of the same size. For example, spatial histograms can be extracted from circular regions with different radiuses.

Many similarity measures for histogram matching have been proposed. In this paper, histogram intersection is used to measure the similarity between two histograms

$$
S_{H I}(\mathbf{H}, \mathbf{S})=\sum_{i=1}^{B} \min \left(H_{i}, S_{i}\right)
$$

where $S_{H I}(\mathbf{H}, \mathbf{S})$ is the histogram intersection statistic with $\mathbf{H}=\left(H_{1}, H_{2}, \cdots, H_{B}\right)^{T}$ and $\mathbf{S}=\left(S_{1}, S_{2}, \cdots, S_{B}\right)^{T}$. Equation (14) is used to calculate the similarity for the nearest neighbor classifier. This measure has an intuitive motivation in that it calculates the common parts of two histograms. Its computational complexity is very low as it requires only simple operations. It should be noted that it is also possible to use other measures such as the chi-square distance [17].

\section{EXTENDing High-ORder LOCAL PATtern to FEATURE IMAGES}

Similar to LBP, the LDP presented in Section II encodes spatially varying patterns in local regions of an image. Conceptually, it is anticipated that extending the proposed high-order local pattern description to feature images containing wider range of appropriate discriminative features could achieve a higher level of system performance. In this section, we investigate the feasibility and effectiveness of extending LDP beyond spatial domain to feature domain. In image processing and object recognition, Gabor features are widely used image feature descriptors extracted by a set of Gabor wavelets (kernels) which model the receptive field profiles of cortical simple cells [5], [6], [15], [28], [29]. They can capture the salient visual properties in an image, such as spatial characteristics, because the kernels can selectively enhance features in certain scales and orientations. Here, we extend LDP to Gabor feature images to enhance the object representation capability. The Gabor wavelets (kernels, filters) can be defined as follows [15]:

$$
\psi_{u, v}(\mathbf{z})=\frac{\left\|\mathbf{k}_{u, v}\right\|^{2}}{\sigma^{2}} e^{\left(-\left\|\mathbf{k}_{u, v}\right\|^{2}\|\mathbf{z}\|^{2} / 2 \sigma^{2}\right)}\left[e^{i \mathbf{k}_{u, v} \mathbf{z}}-e^{-\sigma^{2} / 2}\right]
$$

where $\mathbf{z}=\left(\begin{array}{l}x \\ y\end{array}\right), \mathbf{k}_{u, v}=\left(\begin{array}{c}k_{j x} \\ k_{j y}\end{array}\right)=\left(\begin{array}{c}k_{v} \cos \phi_{u} \\ k_{v} \sin \phi_{u}\end{array}\right), k_{v}=\pi / 2^{(v+2) / 2}$, $\phi_{u}=u(\pi / 8), v=0, \cdots, v_{\max }-1, u=0, \cdots, u_{\max }-1, v$ is the frequency, $u$ is the orientation, $v_{\max }=5, u_{\max }=8$ and $\sigma=2 \pi$.

Let $G_{u, v}(Z)$ denote the Gabor features of an image, where $u$ and $v$ are the orientation and scale of the kernel, respectively. Its $n^{\text {th }}$-order derivatives along $0^{\circ}, 45^{\circ}, 90^{\circ}$, and $135^{\circ}$ directions at $Z=Z_{0}$ can be written as

$$
\begin{aligned}
G_{u, v, 0^{\circ}}^{n}\left(Z_{0}\right) & =G_{u, v, 0^{\circ}}^{n-1}\left(Z_{0}\right)-G_{u, v, 0^{\circ}}^{n-1}\left(Z_{4}\right) \\
G_{u, v, 45^{\circ}}^{n}\left(Z_{0}\right) & =G_{u, v, 45^{\circ}}^{n-1}\left(Z_{0}\right)-G_{u, v, 45^{\circ}}^{n-1}\left(Z_{3}\right) \\
G_{u, v, 90^{\circ}}^{n}\left(Z_{0}\right) & =G_{u, v, 90^{\circ}}^{n-1}\left(Z_{0}\right)-G_{u, v, 90^{\circ}}^{n-1}\left(Z_{2}\right) \\
G_{u, v, 135^{\circ}}^{n}\left(Z_{0}\right) & =G_{u, v, 135^{\circ}}^{n-1}\left(Z_{0}\right)-G_{u, v, 135^{\circ}}^{n-1}\left(Z_{1}\right) .
\end{aligned}
$$

When $n=0, G_{u, v, \alpha}^{0}\left(Z_{0}\right), \alpha=0^{\circ}, 45^{\circ}, 90^{\circ}, 135^{\circ}$ is the same as the original Gabor complex feature $G_{u, v}(Z)$. The real and imaginary parts of the $n^{\text {th }}$-order derivatives $G_{u, v, \alpha}^{n}\left(Z_{0}\right)$ are denoted as $\operatorname{Re}_{G_{u, v \alpha}^{n}\left(Z_{0}\right)}$ and $\operatorname{Im}_{G_{u, v \alpha}^{n}\left(Z_{0}\right)}$, respectively. The $n^{\text {th }}$-order Gabor LDP, $G_{-} L D P_{u, v, \alpha}^{n}\left(Z_{0}\right)$, in $\alpha$ directions at $Z=Z_{0}$ is defined as

$$
\begin{aligned}
& G_{-} L D P_{u, v, \alpha}^{n}\left(Z_{0}\right) \\
&=\{ f\left(\operatorname{Re}_{G_{u, v, \alpha}^{n-1}\left(Z_{0}\right)}, \operatorname{Re}_{G_{u, v, \alpha}^{n-1}\left(Z_{1}\right)}\right) \\
& f\left(\operatorname{Im}_{G_{u, v}^{n-1}\left(Z_{0}\right)}, \operatorname{Im}_{G_{u, v, \alpha}^{n-1}\left(Z_{2}\right)}\right) \\
& f\left(\operatorname{Re}_{G_{u, v, \alpha}^{n-1}\left(Z_{0}\right)}, \operatorname{Re}_{G_{u, v}^{n-1}\left(Z_{3}\right)}\right) \\
& f\left(\operatorname{Im}_{G_{u, v, \alpha}^{n-1}\left(Z_{0}\right)}, \operatorname{Im}_{G_{u, v, \alpha}^{n-1}\left(Z_{4}\right)}\right) \\
& f\left(\operatorname{Re}_{G_{u, v, \alpha}^{n-1}\left(Z_{0}\right)}, \operatorname{Re}_{G_{u, v, \alpha}^{n-1}\left(Z_{5}\right)}\right) \\
& f\left(\operatorname{Im}_{G_{u, v, \alpha}^{n-1}\left(Z_{0}\right)}, \operatorname{Im}_{G_{u, v}^{n-1}\left(Z_{6}\right)}\right) \\
& f\left(\operatorname{Re}_{G_{u, v, \alpha}^{n-1}\left(Z_{0}\right)}, \operatorname{Re}_{G_{u, v, \alpha}^{n-1}\left(Z_{7}\right)}\right) \\
&\left.f\left(\operatorname{Im}_{G_{u, v, \alpha}^{n-1}\left(Z_{0}\right)}, \operatorname{Im}_{G_{u, v, \alpha}^{n-1}\left(Z_{8}\right)}\right)\right\}
\end{aligned}
$$


where $f(\cdot, \cdot)$ is the same binary coding function as defined in Section II. Note that the alternating use of real and imaginary parts from each of the four derivative directions in $G_{-} L D P$ defined in the above equation is for reducing the size of the pattern.

Similar to modeling the gray-level image based $L D P$, the Histogram of Gabor LDP (HGLDP) represented by $H G L D P_{R_{i}}$, at a local region $R_{i}$, is defined as

$$
\begin{aligned}
& H G L D P_{R_{i}}=\left\{H_{G_{-} L D P_{u, v, \alpha}}\left(R_{i}\right) \mid u=0, \cdots, 7\right. \\
& \left.\quad v=0, \cdots, 4 ; i=1, \cdots, L ; \alpha=0^{\circ}, 45^{\circ}, 90^{\circ}, 135^{\circ}\right\}
\end{aligned}
$$

where $H_{G_{-} L D P_{u, v, \alpha}}\left(R_{i}\right)$ is the histogram of LDP extracted from $R_{i}$.

\section{EXPERIMENTS}

A thorough system performance investigation, which covers various conditions of face recognition including lighting, accessory, pose, expression and aging variations, has been conducted. An extensive set of publicly available face databases, FERET [20], CAS-PEAL [7], CMU-PIE [23], Extended Yale B [9], [14], and FRGC [19] databases, were used to evaluate the proposed approach. In the experiments, the facial portion of each original image was normalized and cropped based on the locations of the two eyes. In the following, Experiment A conducts comparative performance evaluations on all the four subsets of the FERET database (all 1,196 people) with expression, lighting and aging variations. Experiment $\mathrm{B}$ reports the experimental results on a subset (the first 300 people) of the CAS-PEAL database with varying accessory, expression and lighting conditions. Experiment $\mathrm{C}$ reports the experimental results on the CMU-PIE database (all 68 people) with pose and illumination variations. Experiment D reports the experimental results on the Extended Yale B database (all 38 people) with severe illumination variations. Experiment $\mathrm{E}$ reports the experimental results on the FRGC database. In all these experiments, we compared the proposed high-order local pattern operator (LDP) with the first-order local pattern operator (LBP) on both gray-level images and Gabor feature images with different parameter settings.

\section{A. Experimental Comparisons on the FERET Database}

The comparative experiments between LDP and LBP were first conducted on the FERET face database, which is widely used to evaluate face recognition algorithms [20]. All the images were normalized and cropped to $88 \times 88$ pixels. We used the same gallery and probe sets as specified in the FERET evaluation protocol. Fa containing 1,196 frontal images of 1,196 subjects was used as the gallery set, while Fb (1,195 images with expression variations), Fc (194 images taken under different illumination conditions), Dup I (722 images taken later in time between one minute to 1,031 days), and Dup II (234 images, a subset of Dup I taken at least after 18 months) were used as the probe sets. To balance the identification accuracy and feature length, we selected the parameters of $4 \times 4$ sized subregions with 8 histogram bins for the gray-level images and $11 \times 11$ sized subregions with 64 histogram bins for the Gabor feature images.

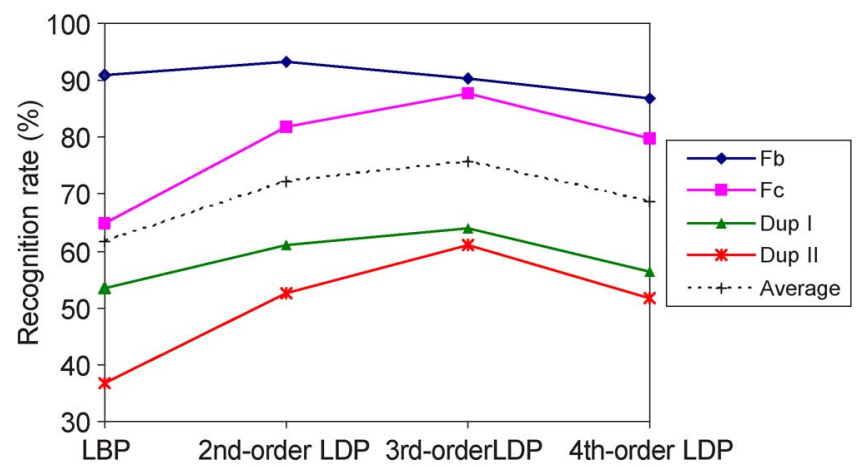

(a)

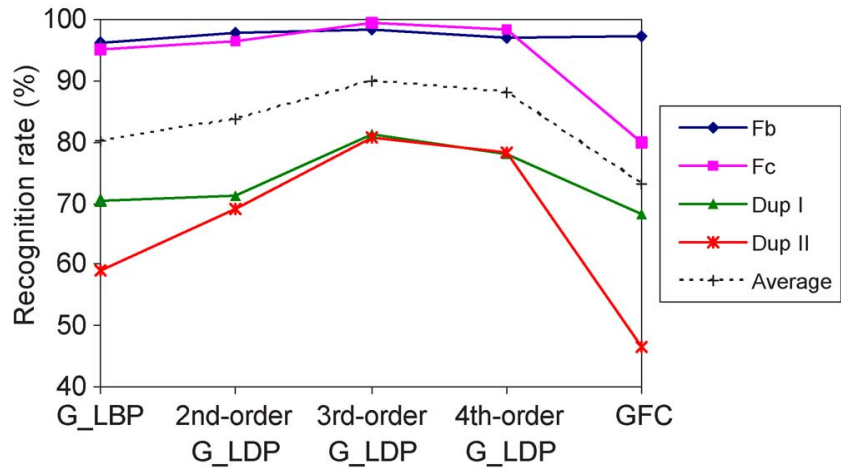

(b)

Fig. 7. Comparative rank-one identification accuracies of LDP and LBP on the FERET database. (a) Results on the gray-level images. (b) Results on the Gabor feature images.

To observe how well LDP performs under different conditions, the experiments were conducted on the individual probe sets. Experimental results in Fig. 7(a) demonstrate that the recognition accuracy in average is significantly improved when the order of local pattern is increased from the first-order local pattern (LBP) to the second-order and the third-order LDPs. Then the performance drops when it reaches to the fourth-order LDP. These results reveal that the high-order local patterns can extract more detailed information than the LBP. The coding function in LDP can alleviate the noise sensitivity problem in the high order derivative images, making LDP more robust and stable in binary encoding identity patterns in human faces. However, it is incapable of dealing with further detailed information contained in the higher-order LDP such as the fourth-order LDP. The results on the large-scale database also show that the detailed information contained in the high-order local patterns can significantly improve the performance of local pattern representation in face recognition.

The experiments were also designed to evaluate the effectiveness of high-order LDP on Gabor feature images. For a fair comparison, LBP was also combined with Gabor real and imaginary parts, named $G_{-} L B P$. Experimental results in Fig. 7(b) illustrate that the third-order $G_{-} L D P$ performs much better than $G \_L B P$. Especially on the Dup subsets, when gallery and probe images are taken at different time with large time intervals of more than one year. It is worth mentioning that the third-order G_LDP method achieves $10.8 \%$ and $21.6 \%$ performance improvements over $G_{-} L B P$ on Dup I and II databases, respectively, showing that the high-order LDP significantly improves 


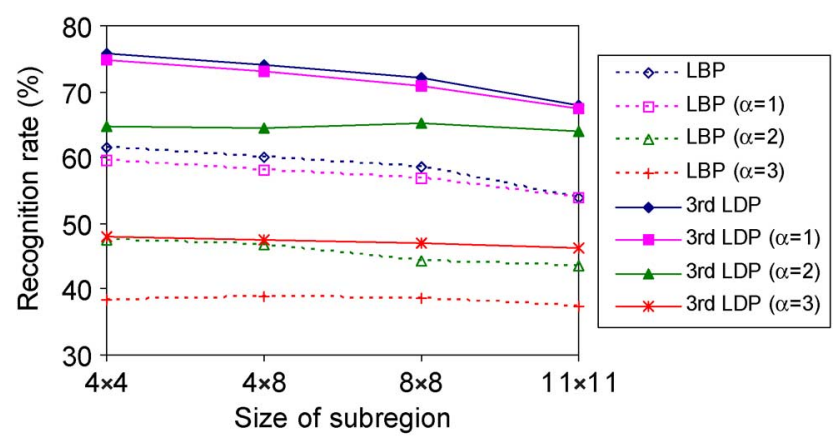

(a)

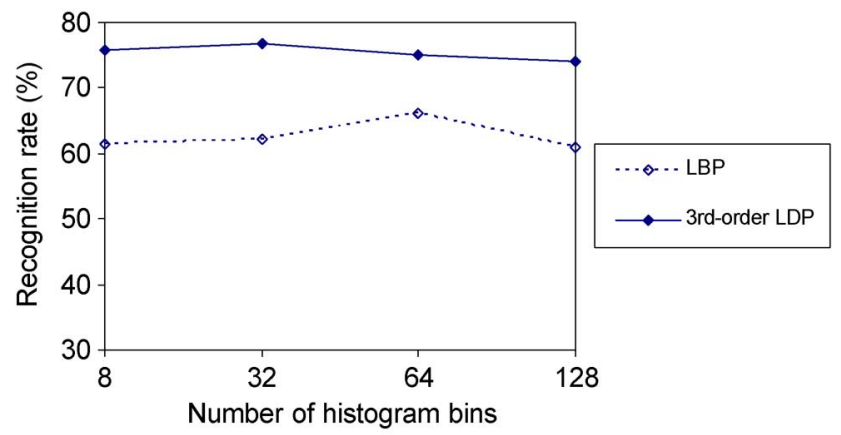

(b)

Fig. 8. Comparative rank-one identification accuracies of LDP and LBP on the FERET gray-level images with different subregion sizes (a) and different histogram bins (b).

the performance of the face recognition system. Fig. 7 reveals that Gabor feature based LDP and LBP have achieved much better performances than the image based LDP and LBP respectively, which confirms that LDP and LBP are both effective on Gabor feature images. Compared to the method of Gabor-Fisher Classifier (GFC) [15] that directly uses Gabor features, $G_{-} L B P$ and $G_{-} L D P$ significantly improve the recognition accuracy.

It is possible that the parameters used in the previous experiments were not finely tuned in favor of LBP. To rule out this possibility, we varied the subregion size and the number of histogram bins and performed the experiments on the gray-level images again. Four different subregion sizes, $4 \times 4,4 \times 8,8 \times 8$, and $11 \times 11$ were tested with the same 8 histogram bins in each subregion. The recognition rates are summarized in Fig. 8(a), showing that an increasing subregion size degrades the system performance due to the loss of spatial information. Still, LDP performs much better than LBP for all these subregion sizes. Then we fixed the subregion size to $4 \times 4$ pixels, and changed the number of histogram bins in each subregion from 128 to 8 using the uniform quantization method that partitions the histogram with equal interval. This reduces the length of the feature vector by 16 times. However, the recognition accuracy curves remain relatively flat for both LBP and LDP in Fig. 8(b), and LDP performs consistently better than LBP for all the testing parameters.

We also evaluated the effect of face misalignment on different subregion sizes of LBP and LDP, respectively. In this experiment, the two eye locations of the FERET probe images were shifted by four independent random values, i.e., displacements of left and right eyes in $x$ and $y$ directions, which were gener-

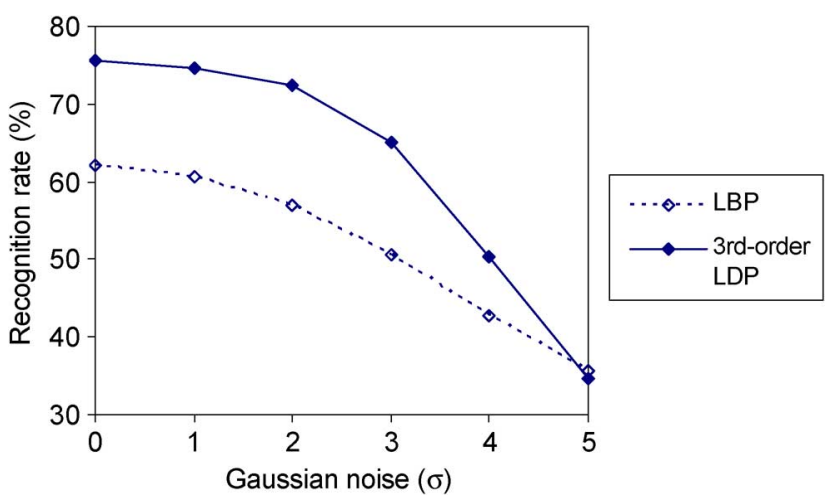

Fig. 9. Comparative rank-one identification accuracies of LDP and LBP on the FERET gray-level images with Gaussian noise of different $\sigma$ added to the probe images.

ated using random Gaussian distribution with $\sigma$ ranging from 1 to 3 by rounding them off to the nearest integers. The eye locations of the face in the FERET gallery set remained unchanged. Fig. 8(a) displays the rank-one identification rates of LBP and LDP with $4 \times 4,4 \times 8,8 \times 8,11 \times 11$ sized subregions and 8 histogram bins. The results demonstrate that the performances of LDP and LBP remain stable to small misalignments $(\sigma=1)$ both for small and large-sized subregions. When $\sigma$ increases further, the identification accuracy curves of both LBP and LDP become flatter, showing that the large subregions are more robust to large misalignments than smaller ones. Note that LDP consistently performs better than LBP in this experiment.

To investigate the sensitivity of LBP and LDP to noise, an experiment was conducted on the same FERET datasets. All the images in the probe sets were added with different levels of Gaussian white noise $(\mu=0, \sigma=1, \cdots, 5)$, while the images in the gallery set remained unchanged. The average recognition rates on the four probe sets against different $\sigma$ of Gaussian noise are illustrated in Fig. 9, showing that LDP maintains a $13.7 \%$ to $15.0 \%$ higher accuracy over LBP when $\sigma$ increases up to 3, and then its accuracy drops greater than that of LBP with larger amount of noise. This is believed due to the coding function in LDP that can alleviate, to certain extent, the noise sensitivity problem in the high-order derivative images.

The computational time for one-to-one image matching (including feature extraction and feature matching) of LBP and the third-order LDP were 0.054 and $0.180 \mathrm{~s}$, respectively. All experiments were conducted on a PC with $3-\mathrm{GHz}$ CPU and 2-GB RAM.

\section{B. Experimental Comparisons on the CAS-PEAL Database}

The CAS-PEAL face database [7] has been publicly released for the purpose of research, which contains 9,060 images of 1,040 subjects. We used the first 300 subjects (indexes from 000001 to 000300) in CAS-PEAL-R1 as the test database. Our gallery set contained 300 faces of the first 300 subjects (one image per person) from the gallery set of CAS-PEAL-R1. The probe set contained 3,109 images of the same 300 subjects from accessory (1,064 pictures), lighting (1,167 pictures) and expression ( 878 pictures) of CAS-PEAL-R1. All the images were normalized and cropped to $88 \times 88$ pixels. 


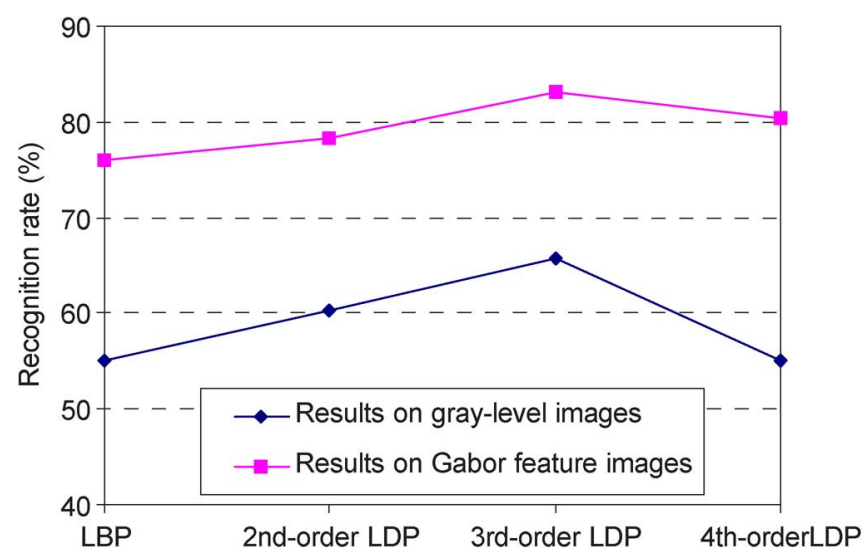

Fig. 10. Comparative rank-one identification accuracies of LDP and LBP on the CAS-PEAL database.

The experiments were first performed to evaluate LDP on the gray-level images using faces with different lighting conditions, expressions, and accessories. Experimental results in Fig. 10 demonstrate that the recognition accuracy is considerably improved when the order of local pattern is increased from the first-order LBP to the second-order and the third-order LDPs. The performance drops back to the level of LBP when it reaches to the fourth-order LDP. These results further confirm that the high-order local patterns can extract more discriminative information than LBP. Note that all the experimental results were obtained with the same parameters of $4 \times 4$ sized subregions and 8 histogram bins for both LDP and LBP.

Fig. 10 also shows the effectiveness of $G_{-} L D P$ on face recognition. Experimental results show that the proposed third-order $G_{-} L D P$ achieves a much better performance than $G_{-} L B P$. Different from the results on the gray-level images, the performance of the fourth-order $G_{-} L D P$ degrades gracefully, probably because Gabor features contain high-order discriminative information and less sensitive to noise due to kernel convolving operation [6], [15]. Compared to the results on gray-level images, the experiment results illustrate that applying local pattern descriptors (LBP and LDP) on Gabor features consistently achieves better performance than directly applying them on the gray-level images, demonstrating that Gabor real and imaginary parts can provide more discriminative information than images. For $G_{-} L D P$ and $G_{-} L B P$ in this experiment, $11 \times 11$ sized subregions with 64 histogram bins were used.

\section{Experimental Comparisons on the CMU-PIE Database}

We further compared the performances between the proposed LDP and LBP under extensive variations in pose and illumination using the CMU-PIE face database [23]. The database contains face images in varying pose, illumination and expression from a total of 68 subjects. In this experiment, the rotated face images in the database that both eyes can be reliably identified for normalization were used as the testing dataset. These images include seven different poses, whose labels are Poses 05, 07, 09, 11, 27, 29, and 37. Each pose has 21 different lighting conditions, labeled as Flashes 2 to 22 . All the images were normalized by aligning the locations of two eyes and cropped to 88

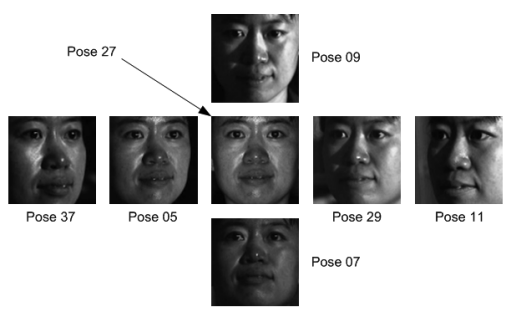

Fig. 11. Samples of the normalized images of one subject from the CMU-PIE database.

$\times 88$ pixels. Fig. 11 shows examples of the normalized images of one subject. The frontal images (Pose 27) with the frontal flash (Flash 08) of each person were used to build the gallery set (one image per person). The remaining images $(21 \times 7-1=$ 146 images per person) were used to create two probe sets for evaluating the proposed method under different range of pose variations. The first probe set contains images of Poses 05, 07, 09,27 , and 29, while the second probe set contains images of Poses $05,07,09,11,27,29$, and 37 . The same parameters as in the previous experiments were used for both LDP and LBP, i.e., $4 \times 4$ sized subregions with 8 histogram bins for gray-level images and $11 \times 11$ sized subregions with 64 histogram bins for Gabor feature images.

Fig. 12 plots the recognition rates of both LDP and LBP on gray-level images and Gabor feature images using the first and second probe sets, respectively. It can be observed from the figure that the second-order and the third-order LDPs obtained higher accuracies than LBP, and the second-order, the third-order and the fourth-order $G_{-} L D P$ s achieved better performances than $G_{-} L B P$ on both two probe sets. On the first probe set [see Fig. 12(a)], the third-order LDP (61.0\%) and the third-order $G_{-} L D P(78.9 \%)$ achieved much higher identification accuracies than LBP $(57.7 \%)$ and $G \_L B P(71.0 \%)$ on gray-level images and Gabor feature images, respectively. The same trend can be observed on the second probe set [see Fig. 12(b)]. Compared with LBP $(41.0 \%)$ and $G_{-} L B P(53$. $5 \%)$, the third-order LDP (43.5\%) and the third-order G_LDP $(62.2 \%)$ obtained the highest rates on gray-level images and Gabor feature images, respectively. Although the performances of both LDP and LBP become worse as the range of pose variation increases from the first probe set to the second probe set, LDP maintains its superiority in recognition accuracy over LBP. These experimental results indicate that the proposed high-order local pattern operator can also effectively extract more discriminative information from face images than LBP under extensive pose and illumination variations. The results also confirm that both LDP and LBP operators can be successfully applied to the Gabor feature images. The performances of local pattern operators on the feature domain are much better than those on the spatial domain.

\section{Experimental Comparisons on the Extended Yale B Database Under Severe Illumination Variations}

The Extended Yale B face database [14] was used to conduct the comparative experiments between the proposed LDP and LBP under severe illumination variations. It contains a total of 38 subjects (including ten subjects from the original Yale B 


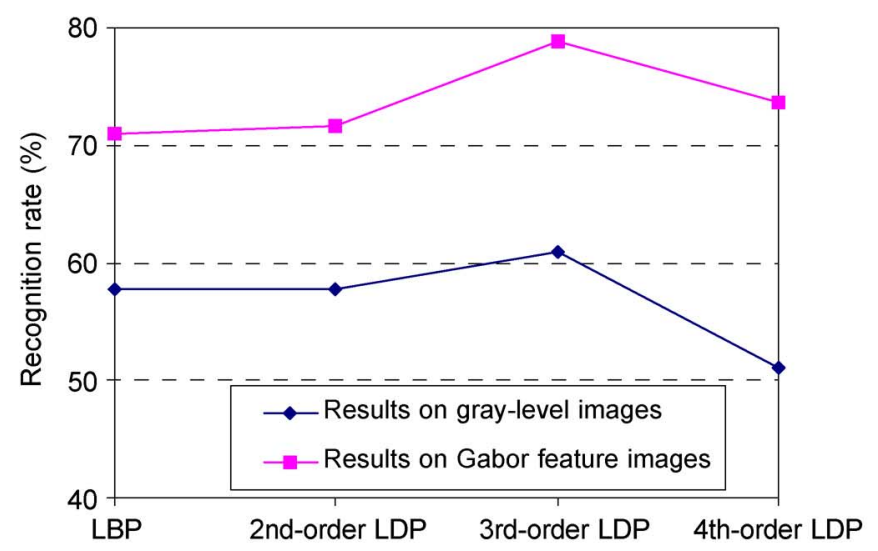

(a)

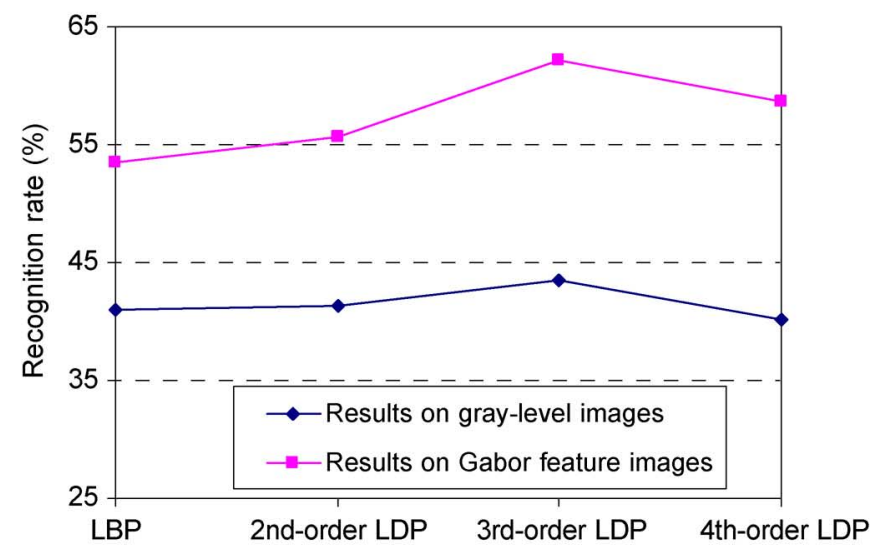

(b)

Fig. 12. Comparative rank-one identification accuracies of LDP and LBP on the CMU-PIE database. (a) Results on the first probe set (images of Poses 05, 07, 09, 27 and 29). (b) Results on the second probe set (images of Poses 05, 07, $09,11,27,29$ and 37).

database [9]) with severe illumination variations. Each subject was imaged under nine poses and 64 different illumination conditions. All the frontal face images with different illuminations have already been manually aligned and cropped to $168 \times 192$ pixels for public downloading from the database website. These aligned frontal face images were used (normalized to $84 \times 96$ pixels) in this experiment. Fig. 13 displays samples of these frontal images of one subject in the database. The frontal face images with lighting direction of $0^{\circ}$ azimuth ("A $\left.+000^{\prime \prime}\right)$ and $0^{\circ}$ elevation ("E +00 ") were used to construct the gallery set (one images per person). All the remaining frontal images were used as the probe set (63 images per person). The parameters of LDP and LBP remained the same as in the previous experiments.

The experimental results of both LDP and LBP on the gray-level images and Gabor feature images are displayed in Fig. 14. Consistent with the results in the previous experiments, the second-order and third-order LDPs and $G_{-} L D P$ s achieved better performances than LBP and $G_{-} L B P$, respectively. Compared with LBP (75.9\%) and G_LBP (86.7\%), the third-order LDP (92.9\%) and the third-order G_LDP (97.9\%) achieved the best performances on gray-level images and Gabor feature images, respectively. These experimental results further demonstrate that the proposed high-order local pattern operator still outperform LBP even with severe illumination
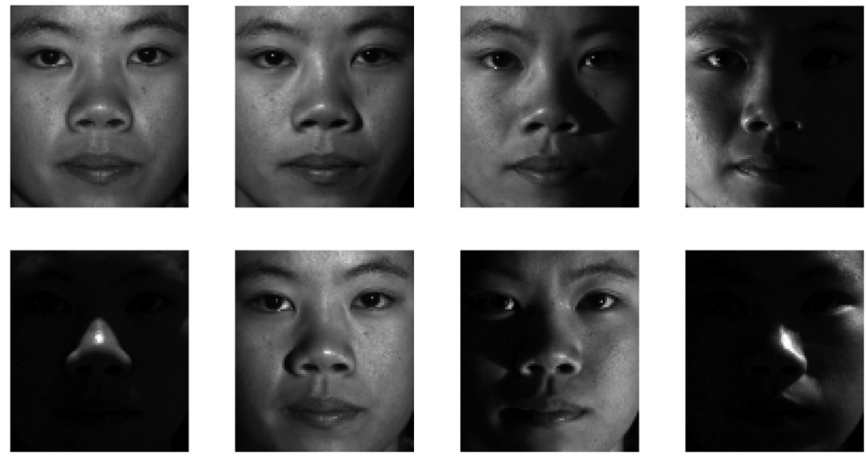

Fig. 13. Samples of the frontal images of one subject from the Extended Yale B database.

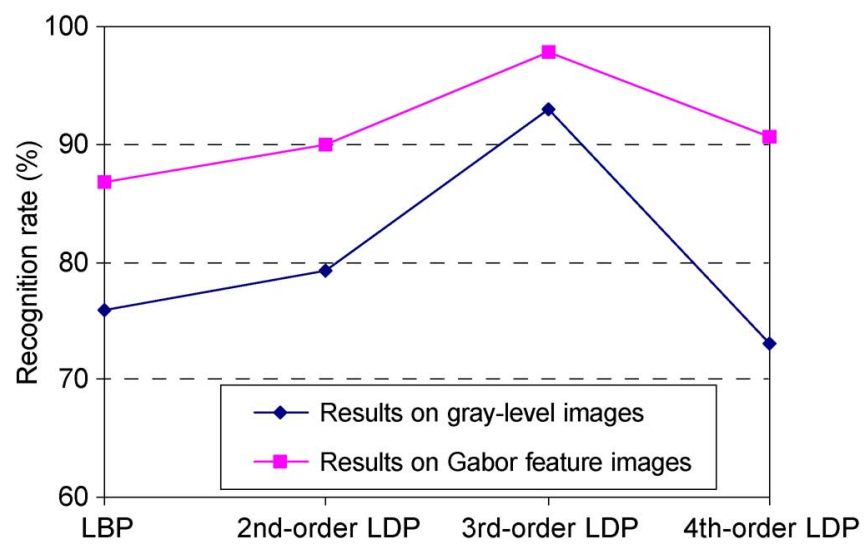

Fig. 14. Comparative rank-one identification accuracies of LDP and LBP on the Extended Yale B database.

variations, demonstrating its effectiveness in extracting more discriminative information from face images.

\section{E. Experimental Comparisons on the FRGC Database}

In order to further evaluate the proposed approach, we also performed experiments on the FRGC version 2.0 database [19]. The FRGC database provides six experimental protocols with the training set. As one of the most challenging protocols, FRGC Experiment 4 involves images with more complex variations such as serious illumination changes, blurring and some occlusions. We selected this protocol in our experiments. The protocol is designed to measure verification performance for 8,014 uncontrolled frontal still images versus 16,028 controlled images. Considering that images in FRGC are of higher resolution, we normalized and cropped them to $128 \times 168$ pixels to evaluate the performances in a higher resolution than in previous experiments. To reduce the feature length, we used $8 \times 8$ sized subregions with 8 histogram bins for both LDP and LBP. Experimental results in Fig. 15 demonstrate that the verification accuracy is improved when the order of local pattern is increased from the first-order local pattern (LBP) to the third-order LDP on both gray-level images and Gabor feature images. These results further indicate that the high-order local pattern can extract more discriminative information than the first-order local pattern.

From above extensive experiments on face recognition, it is noticed that: 1) LDP performs better than LBP on both gray- 


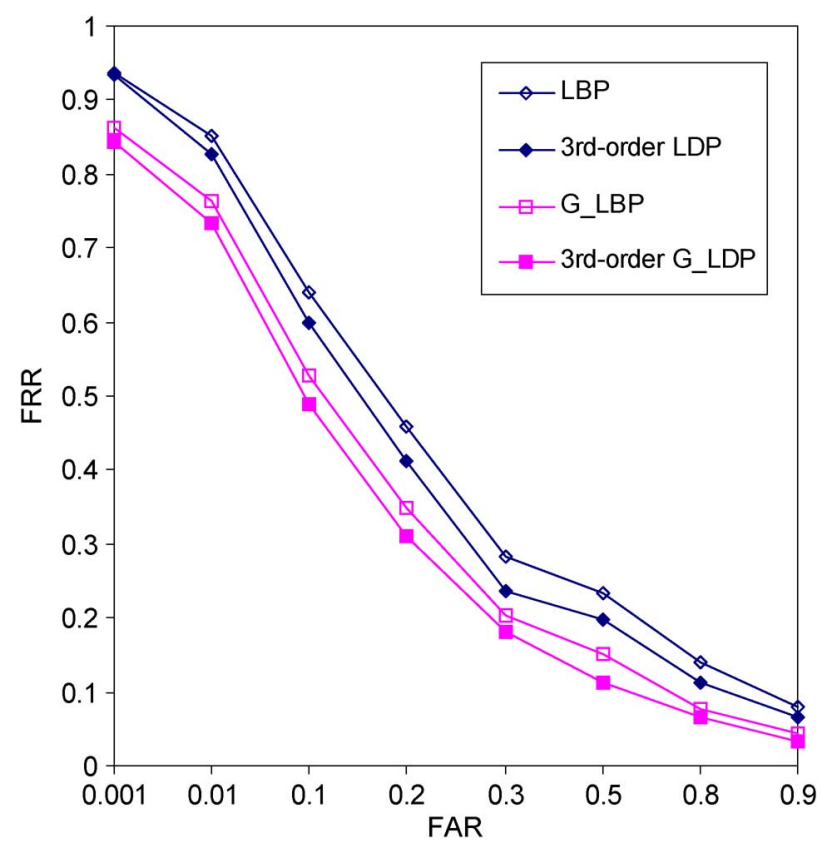

Fig. 15. Comparative verification accuracies of LDP and LBP on the FRGC Experiment 4 database.

level images and Gabor feature images, and for both face identification and face verification. 2) LDP performs superior to LBP and $G_{-} L D P$ performs superior to $G_{-} L B P$ when the order is less than four. 3) The third-order LDP performs the best over all the other order LDPs and LBP on both gray-level images and Gabor feature images. 4) The performance of LDP is largely insensitive to the histogram sampling change. 5) The performance of LDP degrades with noise more strongly than that of LBP but not to a point that can reverse our earlier conclusions.

\section{CONCLUSION AND Future WORK}

This paper investigates the feasibility and effectiveness of using high-order local pattern for face description and recognition. A Local Derivative Pattern (LDP) is proposed to capture the high-order local derivative variations. To model the distribution of LDP micropatterns, an ensemble of spatial histograms is extracted as the representation of the input face image. Face recognition based on LDP can be performed by using histogram intersection as the similarity measurement. Experimental results on an extensive set of face databases, FERET, CAS-PEAL, CMU-PIE, Extended Yale B, and FRGC databases, demonstrate that the proposed high-order local pattern representation outperforms LBP representation in both identification and verification.

The main contributions of this paper include: 1) A novel local descriptor, high-order Local Derivative Pattern, is proposed as an object descriptor. Experiments conducted on various face conditions, including different lightings, expressions, agings, accessories and poses, show that high-order local patterns (LDPs) achieve better performances than the first-order local pattern (LBP). 2) Gabor real and imaginary parts are successfully combined with LDP and LBP. Experimental results show that both LDP and LBP on Gabor feature images achieve much better performance than LDP and LBP on gray-level images.
3) Extensive experiments conducted on various face databases show that the third-order LDP achieves the best performance on both gray-level images and Gabor feature images.

It should be noticed that the high dimensionality of $G_{-} L D P$ is mainly due to the dimensionality of Gabor features, not LDP itself. There are many solutions for dimension reduction, such as linear discriminant analysis (LDA). Investigation and comparison on mutliscale versions of LDP and LBP are interesting future work to fine tune the proposed approach. Due to its excellent performance, we expect that the proposed high-order local pattern descriptor is applicable to other object recognition tasks as well.

\section{REFERENCES}

[1] T. Ahonen, A. Hadid, and M. Pietikäinen, "Face description with local binary patterns: Application to face recognition," IEEE Trans. Pattern Anal. Mach. Intell., vol. 28, no. 12, pp. 2037-2041, Dec. 2006.

[2] M. S. Bartlett, J. R. Movellan, and T. J. Sejnowski, "Face recognition by independent component analysis," IEEE Trans. Neural Netw., vol. 13, no. 6, pp. 1450-1464, Jun. 2002.

[3] P. N. Belhumeur, J. P. Hespanha, and D. J. Kriegman, "Eigenfaces vs. Fisherfaces: Recognition using class specific linear projection," IEEE Trans. Pattern Anal. Mach. Intell., vol. 19, no. 7, pp. 711-720, Jul. 1997.

[4] R. Chellappa, C. L. Wilson, and S. Sirohey, "Human and machine recognition of faces: A survey," Proc. IEEE, vol. 83, no. 5, pp. 705-740, May 1995.

[5] J. G. Daugman, "High confidence visual recognition of persons by a test of statistical independence," IEEE Trans. Pattern Anal. Mach. Intell., vol. 15, no. 11, pp. 1148-1161, Nov. 1993.

[6] D. Gabor, "Theory of communication," J. Inst. Elect. Eng., vol. 93, no. 26, pp. 429-457, 1946.

[7] W. Gao, B. Cao, S. Shan, X. Chen, D. Zhou, X. Zhang, and D. Zhao, "The CAS-PEAL large-scale Chinese face database and baseline evaluations," IEEE Trans. Syst., Man., Cybern. A, Syst. Humans, vol. 38, no. 1, pp. 149-161, 2008.

[8] Y. Gao and M. K. H. Leung, "Face recognition using line edge map," IEEE Trans. Pattern Anal. Mach. Intell., vol. 24, no. 6, pp. 764-779, Jun. 2002.

[9] A. S. Georghiades, P. N. Belhumeur, and D. J. Kriegman, "From few to many: Illumination cone models for face recognition under variable lighting and pose," IEEE Trans. Pattern Anal. Mach. Intell., vol. 23, no. 6, pp. 643-660, Jun. 2001.

[10] F. Goudail, E. Lange, T. Iwamoto, K. Kyuma, and N. Otsu, "Face recognition system using local autocorrelations and multiscale integration," IEEE Trans. Pattern Anal. Mach. Intell., vol. 18, no. 10, pp. 1024-1028, Oct. 1996.

[11] M. Heikkilä and M. Pietikäinen, "A texture-based method for modeling the background and detecting moving objects," IEEE Trans. Pattern Anal. Mach. Intell., vol. 28, no. 4, pp. 657-662, Apr. 2006.

[12] B. Heisele, T. Serre, and T. Poggio, "A component-based framework for face detection and identification," Int. J. Comput. Vis., vol. 74, no. 2, pp. 167-181, 2007.

[13] K. C. Kwak and W. Pedrycz, "Face recognition using an enhanced independent component analysis approach," IEEE Trans. Neural Netw. vol. 18, no. 2, pp. 530-541, Feb. 2007.

[14] K. C. Lee, J. Ho, and D. J. Kriegman, "Acquiring linear subspaces for face recognition under variable lighting," IEEE Trans. Pattern Anal. Mach. Intell., vol. 27, no. 5, pp. 684-698, May 2005.

[15] C. Liu and H. Wechsler, "Gabor feature based classification using the enhanced Fisher linear discriminant model for face recognition," IEEE Trans. Image Process., vol. 11, no. 4, pp. 467-476, Apr. 2002.

[16] T. Ojala, M. Pietikäinen, and D. Harwood, "A comparative study of texture measures with classification based on feature distributions," Pattern Recognit., vol. 29, no. 1, pp. 51-59, 1996.

[17] T. Ojala, M. Pietikäinen, and T. Mäenpää, "Multiresolution gray-scale and rotation invariant texture classification with local binary patterns," IEEE Trans. Pattern Anal. Mach. Intell., vol. 24, no. 7, pp. 971-987, Jul. 2002.

[18] P. S. Penev and J. J. Atick, "Local feature analysis: A general statistical theory for object representation," Network: Comput. Neural Syst., vol. 7, no. 3, pp. 477-500, 1996. 
[19] P. J. Phillips, P. J. Flynn, T. Scruggs, K. W. Bowyer, C. Jin, K. Hoffman, J. Marques, M. Jaesik, and W. Worek, "Overview of the face recognition grand challenge," in Proc. IEEE Computer Society Conf. Computer Vision and Pattern Recognition, 2005, pp. 947-954.

[20] P. J. Phillips, H. Moon, S. A. Rizvi, and P. J. Rauss, "The FERET evaluation methodology for face-recognition algorithms," IEEE Trans. Pattern Anal. Mach. Intell., vol. 22, no. 10, pp. 1090-1104, Oct. 2000.

[21] M. Pietikäinen, T. Ojala, and Z. Xu, "Rotation-invariant texture classification using feature distributions," Pattern Recognit., vol. 33, no. 1, pp. 43-52, 2000.

[22] L. Shen and L. Bai, "Mutualboost learning for selecting Gabor features for face recognition," Pattern Recognit. Lett., vol. 27, no. 15, pp. 1758-1767, 2006.

[23] T. Sim, S. Baker, and M. Bsat, "The CMU pose, illumination, and expression database," IEEE Trans. Pattern Anal. Mach. Intell., vol. 25, no. 12, pp. 1615-1618, Dec. 2003.

[24] M. Turk and A. Pentland, "Eigenfaces for recognition," J. Cogn. Neurosci., vol. 3, no. 1, pp. 71-86, 1991.

[25] O. A. Uwechue and A. S. Pandya, Human Face Recognition Using Third-Order Synthetic Neural Networks. Norwell, MA: Kluwer, 1997.

[26] M. A. O. Vasilescu and D. Terzopoulos, "Multilinear subspace analysis of image ensembles," in Proc. IEEE Computer Soc. Conf. Computer Vision and Pattern Recognition, 2003, vol. 2, pp. 93-99.

[27] L. Wiskott, J. M. Fellous, N. Krüger, and C. von der Malsburg, "Face recognition by elastic bunch graph matching," IEEE Trans. Pattern Anal. Mach. Intell., vol. 19, no. 7, pp. 775-779, Jul. 1997.

[28] B. Zhang, S. Shan, X. Chen, and W. Gao, "Histogram of Gabor Phase Patterns (HGPP): A novel object representation approach for face recognition," IEEE Trans. Image Process., vol. 16, no. 1, pp. 57-68, Jan. 2007.

[29] D. Zhang, W. K. Kong, J. You, and M. Wong, "Online palmprint identification," IEEE Trans. Pattern Anal. Mach. Intell., vol. 25, no. 9, pp. 1041-1050, Sep. 2003.

[30] H. Zhang, W. Gao, X. Chen, and D. Zhao, "Learning informative features for spatial histogram-based object detection," in Proc. IEEE Int. Joint Conf. Neural Networks, 2005, vol. 3, pp. 1806-1811.

[31] G. Zhao and M. Pietikäinen, "Dynamic texture recognition using local binary patterns with an application to facial expressions," IEEE Trans. Pattern Anal. Mach. Intell., vol. 29, no. 6, pp. 915-928, Jun. 2007.

[32] W. Zhao, R. Chellappa, P. J. Phillips, and A. Rosenfeld, "Face recognition: A literature survey," ACM Comput. Surv., vol. 35 , no. 4, pp. 399-459, 2003.

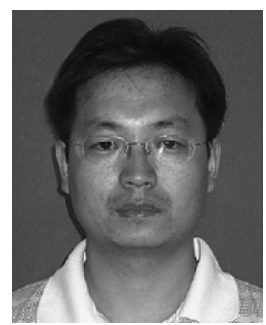

Baochang Zhang received the B.S., M.S., and Ph.D. degrees in computer science from the Harbin Institute of Technology, China, in 1999, 2001, and 2006, respectively.

From 2006 to 2008, he was a Research Fellow with The Chinese University of Hong Kong and Griffith University, Australia. Currently, he is a lecturer with Beihang University, China. His research interests include pattern recognition, machine learning, face recognition, and wavelets.

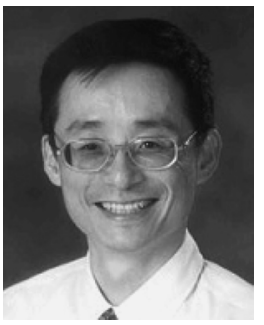

Yongsheng Gao (SM'01) received the B.Sc. and M.Sc. degrees in electronic engineering from Zhejiang University, China, in 1985 and 1988, respectively, and the Ph.D. degree in computer engineering from Nanyang Technological University, Singapore.

Currently, he is an Associate Professor with the Griffith School of Engineering, Griffith University, Australia. He is also with National ICT Australia, Queensland Research Laboratory, leading the Biosecurity group. His research interests include face recognition, biometrics, biosecurity, image retrieval, computer vision, and pattern recognition.

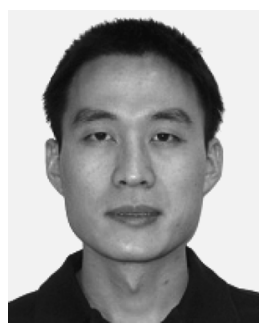

Sanqiang Zhao received the B.E. degree in electronics from Northwestern Polytechnical University, China, in 2000, the M.E. degree in computer science from Beijing University of Technology, China, in 2004, and the Ph.D. degree from Griffith University, Australia, in 2009.

In July 2009, he joined Queensland Research Laboratory, National ICT Australia, as a Researcher. Currently, he is also an adjunct research fellow with the Institute for Integrated and Intelligent Systems, Griffith University. His research interests include pattern recognition, computer vision, face recognition, and image processing.

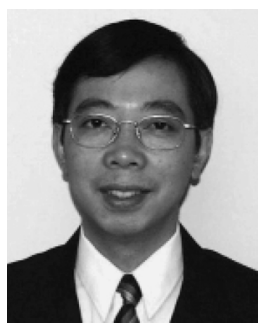

Jianzhuang Liu (M'02-SM'02) received the B.E. degree from Nanjing University of Posts and Telecommunications, China, in 1983, the M.E. degree from Beijing University of Posts and Telecommunications, China, in 1987, and the Ph.D. degree from The Chinese University of Hong Kong in 1997.

From 1987 to 1994, he was a faculty member with the Department of Electronic Engineering, Xidian University, China. From August 1998 to August 2000, he was a research fellow at the School of Mechanical and Production Engineering, Nanyang Technological University, Singapore. Then he was a postdoctoral fellow with the Chinese University of Hong Kong for several years. He is now an Assistant Professor in the Department of Information Engineering, The Chinese University of Hong Kong. His research interests include image processing, computer vision, machine learning, and graphics. 